\title{
Prospective object search in dogs: mixed evidence for knowledge of What and Where
}

\author{
Juliane Kaminski $\cdot$ Julia Fischer $\cdot$ Josep Call
}

Received: 23 August 2006 / Revised: 4 November 2007 / Accepted: 20 November 2007 / Published online: 4 December 2007

(c) Springer-Verlag 2007

\begin{abstract}
We investigated whether two dogs that had been specially trained to retrieve objects by their names were able to integrate information about the identity (What) as well as the location (Where) of those objects so that they could plan their search accordingly. In a first study, two sets of objects were placed in two separate rooms and subjects were asked to retrieve the objects, one after the other. Both dogs remembered the identity of the objects as they reliably retrieved the correct objects. One of the dogs was also able to integrate information about the object's location as he chose the correct location in which the object had been placed. Further investigation of the second dog's behavior revealed that she followed a more stereotyped search strategy. Despite this variation in performance, this study provides evidence for the memory of What and Where in a domestic dog and shows the prospective use of such information in a search task.
\end{abstract}

Keywords Dogs $\cdot$ Spatial cognition $\cdot$ Carnivore . Prospective search $\cdot$ Memory

\section{Introduction}

Foraging is one of the major challenges every animal faces throughout its life. Many animals feed on a variety of resources that are dispersed in space and time, which puts a

J. Kaminski $(\square) \cdot$ J. Call

Max Planck Institute for Evolutionary Anthropology,

Deutscher Platz 6, 04103 Leipzig, Germany

e-mail: kaminski@eva.mpg.de

J. Fischer

Deutsches Primatenzentrum,

Kellnerweg 4, 37077 Göttingen, Germany premium on developing efficient foraging strategies. Animals' foraging efficiency depends on the ability to integrate certain contextual information, for instance the location and the quality of food. To forage more efficiently, animals would highly benefit from remembering, for example, What food sources have been found Where. However, one question, which emerges is how animals store this information. Recent evidence suggests that the cache-recovery behavior of food-storing scrub jays shows many of the characteristics of episodic memory, which was thought to be a uniquely human type of memory (Babb and Crystal 2006; Clayton and Dickinson 1998, 1999b; Griffiths et al. 1999). Following Clayton and Griffiths (2002), episodic memories are not single bits of information; they involve multiple components (What, Where, and When). If these are linked together they entail the creation of a representation of an entire event. However, whether this so-called what, where, when memory system (Suddendorf and Busby 2003a) is equivalent to episodic memory of humans (Tulving 1983) is a currently highly debated issue (Clayton et al. 2003; Suddendorf and Busby 2003b; see also Zentall 2005; Roberts 2002 for critical reviews).

In recent years most researchers have mainly focused on the question of the degree to which different species can integrate memory for object location (Where) and object identities (What). From an evolutionary perspective it is interesting that many omnivorous species have the capacity to remember the What and the Where [rats: Bird et al. (2003), Day et al. (2003); macaques: Hampton et al. (2005), Menzel (1996); apes: Menzel (1999), Scheumann and Call (2006), Schwartz et al. (2005); corvids: Clayton and Dickinson (1999a), Kamil et al. (1994)], and that so far there is no evidence for any member of the order Carnivora to be able to integrate both types of information into a single unit. This may be because carnivores face different ecological 
constraints, which do not require these skills, as for carnivores food does not remain stable and needs to be chased down wherever it is found. However, different carnivores (e.g., dogs and wolves) are known for caching food when they are satiated or when they need to protect their prey from potential scavengers (Peterson and Ciucci 2003). Therefore these species may be able to integrate the Where and What components because after caching food the most successful strategy in finding the food would be to remember what had been hidden where. This makes dogs as a member of the Carnivora a very interesting species to investigate.

There is evidence that dogs can memorize the location of food (Where memory), as dogs can master object displacement tasks - though not invisible displacement tasks (Collier-Baker et al. 2004; Fiset and LeBlanc 2007). However, it is unknown whether dogs can additionally integrate the What component. When confronted with a situation in which they had to retrieve a hidden object on the basis of the place or the features of the hiding location, dogs were shown to rapidly associate spatial cues and follow egocentric search criteria but had difficulties encoding relevant feature information (Dumas 1998). Recently, Kaminski et al. (2004) showed that a domestic dog, Rico, was able to differentiate objects by their labels. Indeed, this dog had an exceptionally good memory for the identities of objects (What memory). However, it is unknown whether this dog would additionally integrate information about the location of the objects.

The aim of the present study was therefore to test whether dogs specially trained to distinguish objects based on their identity would be able to integrate place and identity information to increase their search efficiency by going directly to the location where the requested item was located. In the first study we confronted two dogs with a situation in which they had to search for various objects in two adjacent rooms. Both dogs had been shown to be able to distinguish 170-200 objects (Kaminski et al. 2004; unpublished data). After objects were hidden out of sight of the dogs, subjects were told to fetch the objects one after the other to see whether they remembered the identity and the location of the objects and would use this information to search prospectively. In "study 2" we examined further the search strategies of the less successful dog from "study 1 ".

\section{Study 1}

Methods

\section{Subjects}

Two Border Collies named Rico (male, 9 years old) and Betsy (female, 3 years old) participated in the study. Both dogs had previously been trained to find and retrieve objects by name and were shown to reliably do so under experimental conditions (Kaminski et al. 2004, unpublished data).

\section{Materials}

Objects were mainly children's toys and balls and were all familiar to the dogs. The test took place in three different rooms of the flat of the dog owner. One room served as the dog's starting point during a trial while the other two rooms were where the objects were placed. The dogs could not see the identity of the objects placed until they entered a given room. Upon entering an experimental room they could not see the experimenter, the owner, or the objects placed in the other experimental room. During the trials, the owner was always unaware of the location of the object.

\section{Procedure}

The experimenter selected different objects from the object pool and placed two sets of five (Betsy) or six (Rico) objects in each of the two experimental rooms. The dog stayed with the owner in the start room to ensure that both gathered no information regarding the identity or location of the objects before the trial started. After placing the objects the experimenter returned to the owner and the dog and then the owner asked the dog to fetch an object (Bring xy) designated by the experimenter from a prepared list of all the objects available during the trial. The dog was free to search both rooms and by doing so had the chance to obtain the information about which objects were located in which room. If the dog brought the correct object, it was rewarded with food or play. If it brought the incorrect object or no object at all, the owner asked for the same object again. After bringing an incorrect object or coming back with no object twice, the owner skipped that object and asked for the next one on the list if it was still there. The location of the requested objects was counterbalanced and randomized with the exception that objects were never requested from the same room more than twice in a row. The dog was asked to retrieve objects from the list one after the other with the time interval between two requests being approximately $2 \mathrm{~min}$. All trials were videotaped with three cameras, one in each experimental room and a third one filming the entrance to both experimental rooms to see which room the dog chose first. For each dog we conducted two sessions per day, on two different days. Each session consisted of 10 (Betsy) or 12 (Rico) trials, corresponding to the number of objects available at the beginning of the trial.

We scored the trials from the tapes and analyzed two dependent variables of retrieval accuracy: the number of 
correct objects retrieved (object retrieval) and the location first visited (spatial retrieval). We compared both types of accuracy to the values expected by chance. For the analysis of spatial retrieval we only considered those trials in which the subject brought the correct object. The first trial (corresponding to the first object on the list) was discarded because before it dogs were unaware of the identity of the objects located in each experimental room. In addition, we also discarded the last trial from the object retrieval analyses because there was only one object left.

\section{Results}

We first looked at correct object retrieval. Rico retrieved the correct object in 46 of 48 trials (Binomial test: $P<0.0001)$ whereas Betsy retrieved the correct object in 38 of 40 trials (Binomial test: $P<0.0001$ ). We then looked at spatial retrieval. Overall, Rico directed his first visit significantly above chance to the room where the object was located (proportion of visits to correct room: 29/38, Binomial test: $P=0.002$ ). In contrast, overall Betsy visited the room where the object was located significantly less frequently than expected by chance (proportion of visits to correct room: 9/31, Binomial test: $P=0.029)$.

\section{Discussion}

This study provides evidence that one dog, Rico, clearly integrated information about both object identity (What) and object location (Where), searching the two rooms on the basis of this knowledge. This shows clearly that Rico is able to plan his search in advance and that he uses his knowledge prospectively (e.g., object "xy" is in the kitchen). This ability is comparable to the performance of a language-trained bonobo (Pan paniscus) and children in a similar situation (Savage-Rumbaugh et al. 1993) and is evidence for the memory of What and Where in a domestic dog.

The results of the second dog, Betsy, are hard to interpret. This study suggests that she was using the counterintuitive strategy of searching first where the object was not located which may imply that she was searching prospectively as consistently choosing the wrong room requires as much information about What and Where as consistently getting it right. To investigate Betsy's strategy further we conducted a follow up experiment in which we confronted her with a situation in which encoding was clearly separated from retrieval to see if she would still apply the same search strategy that she used in the present study.

\section{Study 2}

In "study 2" one dog's (Betsy) search strategy was investigated further. To do so we separated the encoding from the retrieval by giving the dog the opportunity to encode both the identity as well as the location of the requested objects before the trial started.

\section{Materials}

The materials were identical to "study 1" except that now the test took place in four different rooms of the flat of the dog owner. One room served as the dog's starting point during a trial while the other three rooms were where the objects were placed.

\section{Procedure}

A single object was placed in each of two of the experimental rooms, while the third room stayed empty. After the placement of the two objects Betsy was given the opportunity to visit all three locations, one after the other, guided by $\mathrm{E}$ on a leash. After that Betsy was guided back to the starting point and requested by the owner, who was unaware of the location of the requested object, to "fetch $\mathrm{xy}$ ". If the dog fetched the correct object the trial ended and a new pair of toys was placed. Betsy received two 12-trial sessions. In the first 12 trials (session 1) the order in which Betsy saw the object locations was kept constant, that is she always was led to room A first, then to room B, then to room C. To investigate whether Betsy's search strategy was biased by the route taken by the experimenter which was always the same, we also presented her with a second set of 12 trials (session 2) during which the order in which the rooms were shown to Betsy was randomized and counterbalanced. Also these rooms were different from those used in session 1.

\section{Results}

Betsy retrieved the correct object in all trials in session 1 and in 11/12 trials in session 2. Betsy consistently directed her first visit to the room where the object was not located in both the first 12 trials, when the order of showing was kept constant (proportion of first visits to correct room: 4/ 12, Binomial test: $P=0.612$ ) and in the second 12 trials, when the order was randomized (proportion of first visits to correct room: 2/12, Binomial test: $P=0.95$ ). In both sessions she had a significant preference for a certain location. In session 1 she preferred to start her search in the kitchen $(10 / 12$, Binomial test: $P<0.01)$ and if she did not find the object proceeded searching in the hall (7/8 Binomial test 
$P<0.01)$. In session 2 she started her search in the office $(10 / 12$ Binomial test $P<0.01)$ and proceeded searching in the entrance $(7 / 10$ Binomial test $P=0.019)$.

\section{Discussion}

This study revealed that Betsy clearly remembered object identity (What) but did not additionally integrate information about object location (Where). Instead she had a strong preference for searching the rooms in a fixed and systematic pattern as she started searching in one particular room and then proceeded to the others.

\section{General discussion}

This study provides evidence that one dog, Rico, can integrate memory of object identity (What memory) with memory of object location (Where memory) and shows that Rico is able to plan his search in advance. The results of the second dog, Betsy, were mixed. The first study suggested that she was using the counterintuitive strategy of searching first where the object was not located. This meant that, unlike Rico, she was less likely to be using a prospective search strategy, but it remained unclear what her strategy actually was. In the second study it became apparent that Betsy was using a more systematic search pattern and did not integrate both types of information, the What and the Where. This shows that the ability to prospectively search integrating What and Where components is within the species' range (Rico) but is not necessarily present in all dogs.

Rico's behaviour constitutes the first evidence for a carnivore to show recalling of the What and Where of an event in an integrated form. One may argue that Rico's abilities in this test are not that surprising given that dogs are known to cache food, most likely an extant behavior of their ancestors, and have also been shown to remember the location where an object had been hidden for up to 4 min (Fiset et al. 2003). However, meat is meat and preying in Carnivores does not necessarily rely on cache-like strategies, therefore the question remains whether it would be at all advantageous for a carnivore to remember what has been stored where. However, like other animals Carnivores apart from food have to locate other valuable resources (e.g., water holes, resting sites). Being able to integrate the What and the Where would surely be beneficial here as it is for finding food. So far there is no evidence that wolves, as dogs' ancestors, have What and Where memory. Therefore one can only speculate about the evolutionary background of this ability in dogs and Rico's behavior in this study may simply be the result of an exceptionally skilled individual.
Rico's special training background alone cannot account for his abilities in the current task as the second dog, Betsy, had the same training background but failed to show the ability to integrate the What and the Where. The use of integrated memory allowed Rico to more efficiently search than the other dog tested in this study. This strategy may be especially important in situations where targets are located very far from each other. Search planning has been described before but here such search took into account the type of object available. Future studies are required to confirm this ability in a larger sample, particularly including other breeds and also including wolves to investigate the evolutionary roots of this behavior.

Acknowledgments We thank the dog owners for their support and time. We are very grateful to Gema Martin-Ordas and Bennett L. Schwartz for helpful comments on an earlier version of this manuscript. The experiments comply with the current laws of the country in which they were performed.

\section{References}

Babb SJ, Crystal JD (2006) Episodic-like memory in rats. Curr Biol 16:1317-1321

Bird LR, Roberts WA, Abroms B, Kit KA, Crupi C (2003) Spatial memory for food hidden by rats (Rattus norvegicus) on the radial maze: studies of memory for where, what, and where. JCP 117:176-187

Clayton NS, Dickinson A (1998) Episodic-like memory during cache recovery by scrub jays. Nature 395:272-274

Clayton NS, Dickinson A (1999a) Memory for the content of caches by scrub jays (Aphelocoma coerulescens). J Exp Psychol Anim Behav 25:82-91

Clayton NS, Dickinson A (1999b) Scrub jays (Aphelocoma coerulescens) remember the relative time of caching as well as the location and content of their caches. JCP 113:403-416

Clayton NS, Griffiths DP (2002) Testing episodic-like memory in animals. In: Squire LR, Schacter DS (eds) Neuropsychology of memory, 3rd edn. Guilford, New York, pp 492-507

Clayton NS, Bussey TJ, Emery NJ, Dickinson A (2003) Prometheus to proust: the case for behavioural criteria for 'mental time traval'. TICS 7:436-437

Collier-Baker E, Davis JM, Suddendorf T (2004) Do dogs (Canis familiaris) understand invisible displacement? JCP 118:421-433

Day M, Lanston R, Morris GM (2003) Glutamate-receptor-mediated encoding and retrieval of paired-associate learning. Nature 424:205-209

Dumas C (1998) Figurative and spatial information and search behavior in dogs (Canis familiaris). Behav Proc 42:101-106

Fiset S, Beaulieu C, Landry F (2003) Duration of dogs' (Canis familiaris) working memory in search for disappearing objects. Anim Cogn 6:1-10

Fiset S, LeBlanc V (2007) Invisible displacement understanding in domestic dogs (Canis familiaris): the role of visual cues in search behavior. Anim Cogn 10:211-224

Griffiths D, Dickinson A, Clayton N (1999) Episodic memory: what can animals remember about their past? TICS 3:74-80

Hampton RR, Hampstead BM, Murray EA (2005) Rhesus monkeys (Macaca mulatta) demonstrate robust memory for what and where, but not when, in an open-field test of memory. Learn Motiv 36:245-259 
Kamil AC, Balda RP, Olson DJ (1994) Performance of four seed-caching corvid species in the radial-arm maze analog. JCP 108:385393

Kaminski J, Call J, Fischer J (2004) Word learning in a domestic dog: evidence for "fast mapping". Science 304:1682-1683

Menzel C (1996) Structure-guided foraging in long-tailed macaques. Am J Primat 38:117-132

Menzel CR (1999) Unprompted recall and reporting of hidden objects by a chimpanzee (Pan troglodytes) after extended delays. JCP 113:426-434

Peterson RO, Ciucci P (2003) The wolf as a Carnivore. In: Mech DL, Boitani L (eds) Wolves. The University of Chicago Press, Chicago

Roberts WA (2002) Are animals stuck in time? Psychol Bull 128:473489
Savage-Rumbaugh E, Murphy J, Sevcik RA, Brakke KE, Williams SL, Rumbaugh DM (1993) Language comprehension in ape and child. Monographs of the Society for Research in Child Development Serial No. 233, vol 58

Scheumann M, Call J (2006) Sumatran orangutans and a yellow-cheeked crested gibbon know what is where. Int J Primat 27:575-602

Schwartz BL, Hoffman ML, Evans S (2005) Episodic-like memory in a gorilla: a review and new findings. Learn Motiv 36:226-244

Suddendorf T, Busby J (2003a) Mental time travel in animals? TICS 7:391-396

Suddendorf T, Busby J (2003b) Like it or not? The mental time travel debate: reply to Clayton et al. TICS 7:437-438

Tulving E (1983) Elements of episodic memory. Clarendon, New York

Zentall TR (2005) Animals may not be stuck in time. Learn Motiv $36: 208-225$ 